\title{
PARK7 protein translocating into spermatozoa mitochondria in Chinese asthenozoospermia
}

\author{
Yi Sun ${ }^{1,2, *}$, Wen-Jia Zhang ${ }^{1,2, *}$, Xin Zhao ${ }^{1,2}$, Ren-Pei Yuan ${ }^{3}$, Hui Jiang ${ }^{3}$ and Xiao-Ping Pu ${ }^{1,2}$ \\ ${ }^{1}$ State Key Laboratory of Natural and Biomimetic Drugs and ${ }^{2}$ Department of Molecular and Cellular Pharmacology, \\ School of Pharmaceutical Science, Peking University, Beijing, People's Republic of China and ${ }^{3}$ Department of \\ Andrology, Peking University Third Hospital, Beijing 100191, People's Republic of China
}

Correspondence should be addressed to X-P Pu; Email: pxp123@bjmu.edu.cn or to

H Jiang; Email: jianghui55@163.com

*(Y Sun and W-J Zhang contributed equally to this work)

\begin{abstract}
PARK7 (DJ1) is a multifunctional oxidative stress response protein that protects cells against reactive oxygen species (ROS) and mitochondrial damage. PARK7 defects are known to cause various physiological dysfunctions, including infertility. Asthenozoospermia (AS), i.e. low-motile spermatozoa in the ejaculate, is a common cause of human male infertility. In this study, we found that downregulation of PARK7 resulted in increased levels of lipid peroxide and ROS, decreased mitochondrial membrane potential, and reduced mitochondrial complex I enzyme activity in the spermatozoa from AS patients. Furthermore, it was observed that PARK7 was translocated into the mitochondria of damaged spermatozoa in AS. Finally, we examined the oxidative state of PARK7 and the results demonstrated the enhancement of oxidation, expressed by increased sulfonic acid residues, the highest form of oxidation, as the sperm motility decreased. Taken together, these results revealed that PARK7 deficiency may increase the oxidative stress damage to spermatozoa. Our present findings open new avenues of therapeutic intervention targeting PARK7 for the treatment of AS.

Reproduction (2014) 148 249-257
\end{abstract}

\section{Introduction}

Asthenozoospermia (AS), defined as low-motile spermatozoa in the ejaculate, is a frequent cause of human male infertility (Chemes et al. 1998). It is reported that more than $80 \%$ of the spermatozoa from infertile patients had motility defects and $19 \%$ of them were diagnosed with AS (Curi et al. 2003). Oxidative stress is one of the main issues associated with AS. When the physiological equilibrium between reactive oxygen species (ROS) production and elimination is perturbed, spermatozoa function may be compromised, and indeed oxidative stress is associated with male infertility (Sikka et al. 1995). Increased ROS levels have been implicated in spermatozoa DNA fragmentation, decreased motility, lipoperoxidation damage, and increased apoptosis (Agarwal et al. 2008). It has been proposed that the mitochondria are a major contributor to the oxidative stress followed by defective human spermatozoa (Koppers et al. 2008). Electron microscopy revealed that spermatozoa from AS patients have disordered mitochondria, significantly shorter midpieces, and fewer mitochondrial gyres when compared with their normozoospermic counterparts (Mundy et al. 1995). Furthermore, spermatozoa quality, particularly motility, is positively correlated with the enzymatic activities of the electron transfer chain (ETC) complexes (Ruiz-Pesini et al. 1998, 2000) and the expression of ETC subunits (Amaral et al. 2007). The mitochondria are continuously protected against ROS damage by various antioxidants and multiple enzymatic defense systems, including Superoxide Dismutase, catalase, the glutathione peroxidase/reductase system, and non-enzymatic substances, such as ascorbic acid, glutathione, and a-tocopherol (Mannella 2008).

PARK7 (DJ1) is ubiquitously expressed in various human tissues, with a particularly high level of expression in the testes (Nagakubo et al. 1997). Although its precise biochemical function is not fully known, accumulated studies demonstrated that PARK7 is a multifunctional protein and plays essential roles in tissues with higher order biological functions, including cell transformation (Nagakubo et al. 1997), sperm maturation and fertilization (Klinefelter et al. 1997, Ooe et al. 2005, Hao et al. 2010), oxidative stress response (Mitsumoto \& Nakagawa 2001, Mitsumoto et al. 2001, Kinumi et al. 2004, Taira et al. 2004), and Parkinson's disease (Bonifati et al. 2003). PARK7 was initially identified as a novel oncogene product that transforms NIH3T3 cell in cooperation with H-Ras 
(Nagakubo et al. 1997). Around the same time, PARK7 and its homologues, sperm protein 22 (SP22) and contraception-associated protein 1 , were identified as the first proteins to be correlated with male infertility (Klinefelter et al. 1997, Wagenfeld et al. 1998, Welch et al. 1998). In a study conducted on rats, it was reported that the amount of PARK7 in spermatozoa and the efficiency of fertilization decreased with an increasing dose of spermatozoa toxicant such as ornidazole and epichlorohydrin, to which the rats were exposed (Wagenfeld et al. 1998, Welch et al. 1998). It was also demonstrated that PARK7 began to be expressed after the appearance of a second spermatocyte during spermatogenesis and is finally located in the sperm head, implying an important function of PARK7 in spermatogenesis (Wagenfeld et al. 1998, Welch et al. 1998). Then it was found that an anti-SP22/DJ1 antiserum abrogated the fertilization activity of sperm (Klinefelter et al. 2002, Okada et al. 2002). Importantly, PARK7 can protect cells against oxidative stress damage, in which ROS was eliminated in vitro and in vivo by oxidizing PARK7 itself (Yokota et al. 2003, Taira et al. 2004). PARK7 possesses three cysteines at amino acid positions 46, 53, and 106 (Cys46, Cys53, and Cys106). Oxidation of Cys106 is essential for PARK7 to exert its full activities (Takahashi-Niki et al. 2004), but unoxidized PARK7 and oxidized PARK7 in the sulfonic $\left(\mathrm{SO}_{3} \mathrm{H}\right)$ form are both inactive (Yokota et al. 2003). PARK7 is mainly located in the nucleus and cytoplasm (Nagakubo et al. 1997, Shinbo et al. 2006). When cells were treated with an inducer of ROS, PARK7 was translocated into the mitochondria for maintenance of mitochondrial complex I (Cl) activity and was co-localized with ND1 and NDUFA4, subunits of mitochondrial $\mathrm{Cl}$ encoded by mitochondrial and nuclear DNA respectively. This oxidation-induced mitochondrial relocalization of PARK7 and protection against cell death were abrogated in the mutating cysteine residue Cys106A but not Cys53A or Cys46A (Canet-Aviles et al. 2004). Furthermore, the $\mathrm{Cl}$ activity was reduced in two types of PARK7-knockdown NIH3T3 and HEK293 cells (Hayashi et al. 2009).

Although PARK7 has been reported to be related to infertility of rats and mice and to participate in fertilization for the spermatozoa to penetrate into the zonae pellucidae of eggs (Welch et al. 1998, Klinefelter et al. 2002, Okada et al. 2002, Yoshida et al. 2003), the characteristics of PARK7 in spermatozoa from AS patients are still not clear, and the function of mitochondria-resident PARK7 is still not known.

In this study, we analyzed the expression of PARK7 in human spermatozoa and its effect on mitochondrial function. The results showed that downregulation of PARK7 in spermatozoa from AS patients depressed the mitochondrial function and that PARK7 was translocated into the mitochondria.

\section{Materials and methods}

\section{Patients}

From June 2012 to October 2013, we enrolled 150 males (aged 21-45 years) diagnosed with infertility in the Department of Andrology, Peking University Third Hospital, Beijing, China, controlled by 86 age-matched control subjects with normal semen parameters (Supplementary Table 1, see section on supplementary data given at the end of this article). Semen samples of patients with pyospermia, varicocele, or smoking history were not eligible. According to the severity of AS, patients were divided into two groups: mild AS patients with A-grade spermatozoa percentage $>10 \%(n=81)$ and moderate AS patients with A-grade spermatozoa percentage $<10 \%(n=69)$. All participants provided informed consent for participation in the study approved by the Ethics Institutional Review Board of Peking University Third Hospital.

\section{Semen analysis}

Seminal samples were collected by masturbation after 2-7 days of sexual abstinence. Each semen sample was liquefied for $30 \mathrm{~min}$ at $37^{\circ} \mathrm{C}$ before sample preparations. The volume, $\mathrm{pH}$, and spermatozoa concentration and motility of sample were assessed according to the World Health Organization guidelines (Organization 2010) by a computer-assisted semen analyzer (Weili Company, Beijing, China).

\section{Total spermatozoa protein extraction}

After analysis of semen parameters, the total spermatozoa protein was extracted as described previously (An et al. 2011). In brief, the remaining semen was washed twice with Hanks balanced salt solution by centrifugation. The combined spermatozoa pellets, resuspended in $80 \mathrm{mM}$ Tris- $\mathrm{HCl}$ buffer $(\mathrm{pH} 7.4)$, were oscillated using an ultrasonic apparatus and then homogenized for $1 \mathrm{~h}$ on ice. Finally, the mixtures were centrifuged to remove the insoluble debris, and the supernatant fraction was frozen at $-80^{\circ} \mathrm{C}$ until use.

\section{Western blotting}

The concentration of total protein in the spermatozoa lysates was measured using a Bradford reagent (Cellchip Biotechnology Co., Ltd, Beijing, China). Proteins (20 mg) were separated by $12.5 \%$ SDS-PAGE and transferred onto a PVDF membrane (Millipore, Billerica, MA, USA). The membrane was blocked with $5 \%$ non-fat milk in Tris-buffered saline solution with Tween 20 with agitation. Proteins were sequentially incubated with the anti-DJ1 MAB (Abcam, Cambridge, UK) and HRP-conjugated IgG (KPL, Gaithersburg, MD, USA). Western Blotting Substrate (Applygen Technologies, Beijing, China) was used for the detection of ECL on an X-ray film. GAPDH was used as an internal standard. For quantification of PARK7 expression levels, Quantity One Software (Bio-Rad) was used to quantify the optical density of each band. Levels of PARK7 were normalized against GAPDH. 


\section{Lipid peroxide assay}

Lipid peroxidation (LPO) was measured using the thiobarbituric acid (TBA) assay according to the method described previously (Searle \& Willson 1983) with some modifications. Spermatozoa total protein of $200 \mathrm{mg}$ was treated with $4 \mu \mathrm{l}$ of $0.01 \mathrm{M}$ cysteine and $8 \mu \mathrm{l}$ of $0.001 \mathrm{M} \mathrm{FeSO}_{4}$. After the mixture was incubated, $100 \mu \mathrm{l}$ of $20 \%$ TCA along with a chromogenic agent of $100 \mu \mathrm{l}$ of $0.1 \%$ TBA was added to terminate the reaction. The TBA reactive substance - MDA in the resultant mixture - was measured by the absorbance at $532 \mathrm{~nm}$.

\section{ROS assay}

The level of ROS in spermatozoa was quantified using the ROS assay kit. Spermatozoa were washed with Biggers, Whitten, and Whittingham medium (BWW; Organization 2010), suspended in DCFH-DA diluted to a final concentration of $10 \mathrm{mM}$ at a density of $1 \times 10^{6}$ spermatozoa $/ \mathrm{ml}$, and incubated in the dark. After being washed three times with PBS, the fluorescence was analyzed quantitatively by flow cytometry (BD Biosciences, Franklin Lakes, NJ, USA). The excitation and emission wavelengths were set at 488 and $525 \mathrm{~nm}$.

\section{Indirect immunofluorescence}

Spermatozoa were collected by density gradient centrifugation (WHO 2010). Then, $1 \mathrm{ml}$ of semen was layered on the density gradient media composed of $1 \mathrm{ml}$ of $40 \%(\mathrm{v} / \mathrm{v})$ Percoll solution (Sigma) above $1 \mathrm{ml}$ of $80 \%$ (v/v) Percoll solution. All Percoll solutions were buffered with BWW solution. Following centrifugation, the spermatozoa accumulated at the bottom of the tube were collected.

An aliquot of spermatozoa washed with Percoll solution and resuspended in PBS was spotted on glass slides and air-dried. These preparations were incubated with $200 \mathrm{nM}$ MitoTracker Deep Red FM (MT-DR FM; Invitrogen), fixed with 4\% paraformaldehyde, and then permeabilized with ice-cold acetone, according to the operation manual recommended by Life Technologies Corporation. These preparations were blocked with $10 \%$ goat serum, and then sequentially incubated with the anti-DJ1 MAB (Abcam) or normal rabbit IgG as a negative and FITC-conjugated anti-rabbit antibody (Proteintech, Chicago, IL, USA). The nuclei were counterstained with Hoechst 33342 and mounted with antifade mounting medium. All samples were observed under a laser scanning confocal microscope (Leica, Mannheim, Germany).

\section{Flow cytometric analysis}

The spermatozoa were mixed gently in $200 \mathrm{nM}$ MT-DR FM solution and incubated for $45 \mathrm{~min}$ in the dark. After staining was complete, the spermatozoa were repelleted by centrifugation and resuspended in fresh prewarmed BWW solution. Their fluorescence was measured using a FACSCalibur flow cytometer (BD Biosciences) with excitation at $644 \mathrm{~nm}$ and emission at $665 \mathrm{~nm}$. The amount of fluorescence was also quantified using the 'CELL QUEST' program with a value of 'median'.

\section{Measurement of mitochondrial Cl activity}

The mitochondrial $\mathrm{Cl}$ activity was determined by spectrophotometric assay using a Quantitative Detection Kit of mitochondrial respiratory chain $\mathrm{Cl}$ activity (Genmed Scientifics, Inc., Wilmington, DE, USA). According to the provided protocol, $50 \mu \mathrm{g}(25 \mu \mathrm{l})$ of total proteins isolated from spermatozoa and $25 \mu$ l Reagent C (negative control) were treated with Reagent A (buffer), B (reaction solution), and D (substrate solution). The absorbance was measured at 0 and $5 \mathrm{~min}$ at a wavelength of $340 \mathrm{~nm}$. The values were corrected with nonspecific absorption at $380 \mathrm{~nm}$.

\section{Immunoprecipitation and mass spectrometry analysis}

The oxidative state of Cys106 of PARK7 was identified by LTQ Orbitrap mass spectrometry analysis as described previously (Gao et al. 2011) with some modifications. The spermatozoa proteins were concentrated by centrifugation in ultrafiltration tubes (Millipore) at about $8 \mu \mathrm{g} / \mu \mathrm{l}$. The proteins $(3.5 \mathrm{mg}$ ) were immunoprecipitated with a rabbit anti-DJ1 polyclonal antibody and separated on $20 \%$ polyacrylamide gel. After the gel had been stained with Coomassie brilliant blue, the band corresponding to PARK7 was excised for measurement by LTQ Orbitrap Velos Pro (Thermo, Waltham, MA, USA) mass spectrometry.

\section{Statistical analysis}

Data are expressed as means \pm s.D. Statistical analysis was performed using a one-way ANOVA for different groups of measurements. Comparisons with $P<0.05$ were generally considered to be significant although $P<0.01$ was also given for those comparisons with more significant difference as indicated in figures.

\section{Results}

\section{Decrease in spermatozoa PARK7 expression in AS}

PARK7 was detected as a single band with a molecular weight of $\sim 20 \mathrm{kDa}$ by western blotting (Fig. 1A). A significant difference in the expression level of PARK7 was demonstrated between AS and normal spermatozoa $(P<0.001$; Fig. 1B), and the positive relationship between the decrease in PARK7 and the severity of AS was revealed by the Kruskal-Wallis Test (Fig. 1C).

\section{Increase in LPO and ROS in AS}

Two indicators, LPO and ROS, were selected as evaluation indexes of the spermatozoa peroxide level. Thiobarbituric acid reactive substances (TBARS) content is an index of LPO and is usually assayed for indicating oxidative damage to plasma membranes. The data reported in Fig. 2A indicate that the TBARS content increased significantly in spermatozoa from AS patients. As shown in Fig. 2B, there is a considerable increase in the intracellular ROS production in AS spermatozoa compared with the normal spermatozoa by flow 
A
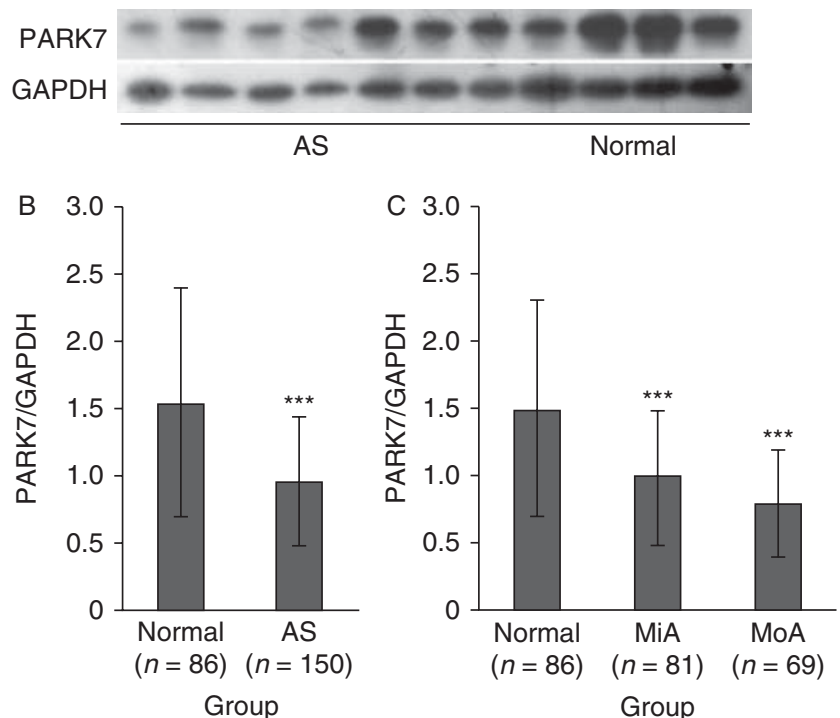

Figure 1 The expression level of PARK7 was analyzed by western blotting. (A) Before considering the severity of AS, seven bands on the left side represent $A S$, while the other four represent the normal group. GAPDH was used as a loading control; (B) histogram showing the quantification of the expression level of each sample using GAPDH as a loading control before considering the severity of AS. Significant differences are shown between the two groups; and (C) The samples of AS were divided into two subgroups in line with the percentage of A-grade sperm: MiA and MoA. The results shown in the histogram indicate that the expression level of PARK7 decreased more aggressively with exacerbation of AS. All data are expressed as mean \pm s.D. $n$, number of samples used. ${ }^{* * *} P<0.001$ vs normal group by the Kruskal-Wallis test. AS, asthenozoospermia; MiA, mild asthenozoospermia; MoA, moderate asthenozoospermia.

cytometry. In fact, a lower percentage of spermatozoa with low levels of ROS was detected, along with a concomitant higher percentage of those with high levels of ROS.

\section{Immunocytochemical localization of PARK 7 in spermatozoa in AS}

Preliminary experiments showed that PARK7 was translocated into the midpiece of spermatozoa, where the mitochondria exist (Supplementary Fig. 1, see section on supplementary data given at the end of this article). To indicate the location of the mitochondria, spermatozoa were incubated with MT-DR FM, which passively diffuses across the plasma membrane and accumulates in the active mitochondria. Spermatozoa were also treated with the anti-DJ1 MAB and then with a FITC-conjugated anti-rabbit IgG, and the nuclei were counterstained with Hoechst 33342. These reactions were represented by red, green, and blue fluorescence respectively, and these images were merged.

From the imaging results, we found that PARK7 was visualized as a scarf-like structure in the damaged spermatozoa midpiece, in randomly selected areas. Moreover, the more serious the disease, the greater the number of 'scarfs', and there was nearly no scarf in the normal spermatozoa (Fig. 3).

The intensities of red fluorescence from the excited MT-DR FM corresponding to the levels of mitochondrial membrane potential (MMP) showed that the MMP of spermatozoa gradually disappeared with the increasing severity of AS.

\section{MMP analysis by flow cytometry}

In this study, the mitochondria of spermatozoa were incubated with MT-DR FM and measured using flow cytometry. By doing so, we were able to isolate two different subpopulations: MT-DR FM-positive and -negative spermatozoa (Fig. 4A and B). The percentage of MT-DR FM-positive and -negative spermatozoa was distinct in the three groups $(48.7 \pm 15.87$ and $51.35 \pm 15.9 \%$ for normal samples, $43.25 \pm 20.45$ and $56.81 \pm 20.46 \%$ for mild AS, and $21.24 \pm 12.15$ and $78.89 \pm 12.22 \%$ for moderate AS; Fig. 4C). The moderate AS group contained the least MT-DR FM-positive and the most MT-DR FM-negative spermatozoa $(P<0.01)$, which suggested that the percentage of MT-DR FM-positive spermatozoa of a given sample was positively correlated with its quality.

Another distinctive feature of each group, namely the mean fluorescence intensity, was observed. Especially in the MT-DR FM-negative spermatozoa, the fluorescence
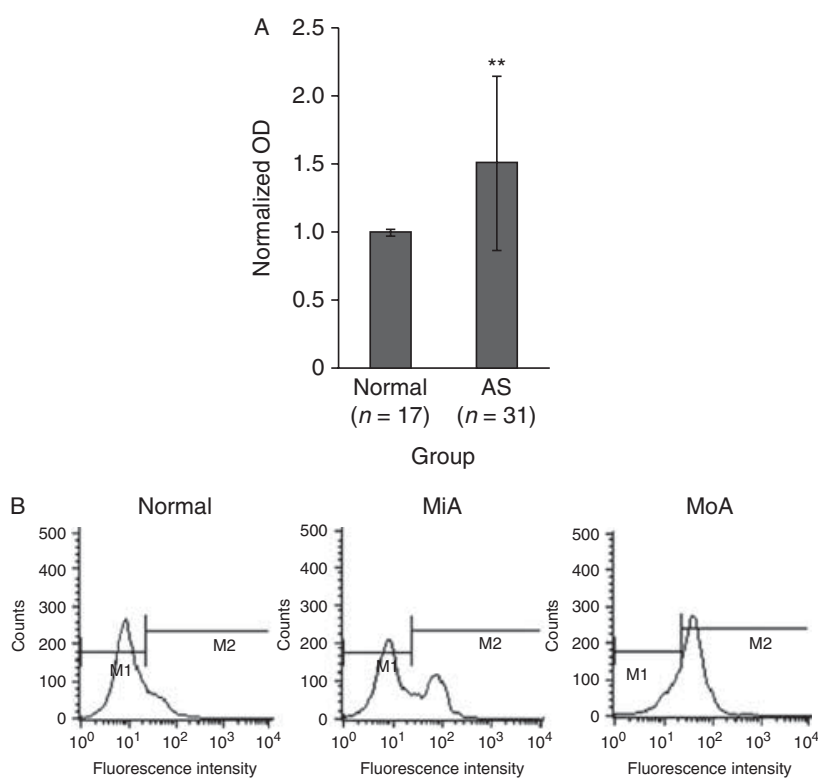

Figure 2 Increase in LPO and ROS in AS. (A) The ordinate value represents the normalized OD against the mean OD of normal group. Significant enhancement of lipid peroxide in AS compared with normal group was shown. All data are expressed as mean \pm s.D. $n$, number of samples used. ${ }^{* *} P<0.01$ vs normal group by the Kruskal-Wallis test and (B) flow cytometry analysis of reactive ROS in sperms. It was demonstrated that along with the aggravation of AS, the formation and accumulation of ROS obviously increased. AS, asthenozoospermia; MiA, mild asthenozoospermia; MoA, moderate asthenozoospermia. 


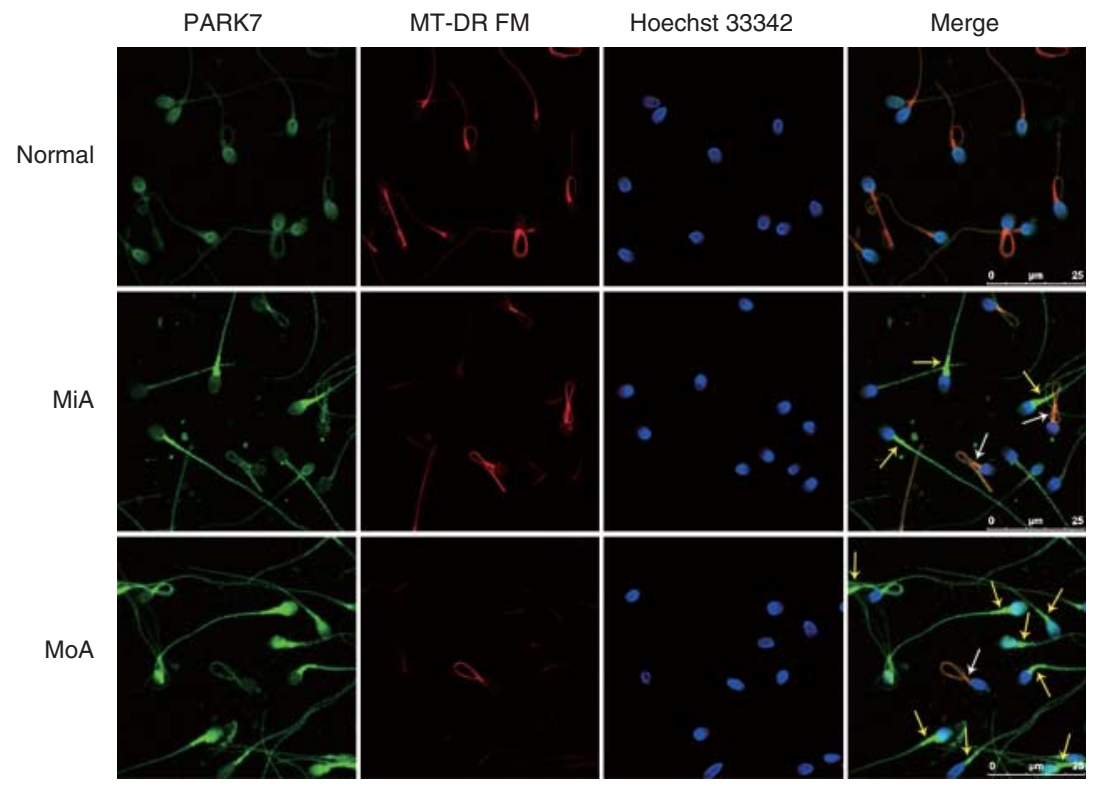

Figure 3 Immunofluorescence localization of PARK7 and mitochondria in ejaculated spermatozoa. Spermatozoa were treated with MT-DR FM (red) (as described in 'Materials and methods' section), and then spermatozoa were immunostained with an anti-DJ1 antibody (green). The nuclei were counterstained with Hoechst 33342 (blue). The images were merged (merge). Yellow arrows indicate the depolarized mitochondria and white arrows represent the electrochemically active mitochondria that were permeated with MT-DR FM in the MiA or MoA merge. PARK7, resembling a 'scarf', appeared in the depolarized mitochondria (yellow arrows) and was then visualized using a confocal laser microscope. Scale bars $=25 \mu \mathrm{m}$. MiA, mild asthenozoospermia; MoA, moderate asthenozoospermia; MT-DR FM, MitoTracker Deep Red FM.

intensity exhibited significant differences among these three groups $(3.53 \pm 0.86 \%$ for normal samples, $4.00 \pm$ $0.52 \%$ for mild AS samples, and $4.55 \pm 0.61 \%$ for moderate AS samples; Fig. 4D). It is interesting that in moderate AS, the fluorescence intensity of the MT-DR FM-negative spermatozoa was highest compared with normal and mild samples $(P<0.01)$. This finding indicated that the mitochondrial function of these spermatozoa was improved to a certain extent via some unknown compensatory mechanisms.

\section{Impairment of mitochondrial $\mathrm{Cl}$ activity}

Mitochondrial $\mathrm{Cl}$ is the first catalytic system in the respiratory chain. Specific reduction in mitochondrial $\mathrm{Cl}$ activity inhibited sperm motility through regulation of the $\mathrm{NAD}^{+} / \mathrm{NADH}$ redox balance. In this study, fresh ejaculated semen samples were collected, from which the total protein of sperm was isolated, and the $\mathrm{Cl}$ enzyme activity was measured. In comparison to normal samples, $\mathrm{Cl}$ enzyme activity was significantly reduced in AS samples and showed a positive correlation as the severity of AS paralleled the PARK7 expression (Fig. 5).

\section{Cys106 oxidation modification of PARK7 in spermatozoa of $A S$}

PARK7 contains three cysteine residues (Cys46, Cys53, and Cys 106) whose oxidation states regulate its function. Of these, Cys106 is particularly susceptible to gradual oxidation to sulfenic acid $(\mathrm{SOH})$, sulfinic acid $\left(\mathrm{SO}_{2} \mathrm{H}\right)$, and finally sulfonic acid $\left(\mathrm{SO}_{3} \mathrm{H}\right)$. The function of PARK7 is closely related to its oxidation state, which may cause the inactivation of PARK7. We observed Cys106 in the sequence of sperm PARK7, which was obtained by immunoprecipitation followed by SDS-PAGE. The percentage of each form was used to reflect the oxidative state of PARK7. As shown in Fig. 6, the value of oxidized Cys106, especially the $\mathrm{SO}_{3} \mathrm{H}$ form, has been shown to intensify in PARK7 proteins derived from sperms of AS compared with the normal ones.

\section{Discussion}

This study has revealed a new molecular mechanism underlying PARK7-mediated human spermatozoa motility by protecting the mitochondrial function. We demonstrated that PARK7 expression was reduced in ejaculated spermatozoa from AS patients compared with normal donors. We also found that the content of TBARS-MDA in spermatozoa was significantly increased in AS patients whose PARK7 expression was blocked using TBA and that the decreased expression of PARK7 was consistent with the level of MMP. Importantly, we confirmed that PARK7 was translocated into the mitochondria and improved the activity of $\mathrm{Cl}$ against oxidative stress. Finally, we analyzed the oxidation state of the three cysteine residues of PARK7, Cys46, Cys53, and Cys106, and the results demonstrated that the level of superfluous oxidation was increased in AS patients.

\section{Repression in PARK7 expression in AS diminishes the antioxidant capacity of spermatozoa}

Spermatozoa are particularly vulnerable to oxidative stress because of their characteristics, high polyunsaturated fatty acid content, intrinsic deficiencies of intracellular antioxidant enzymes, and limited capacity for DNA repair (Talevi et al. 2013). Under physiological 


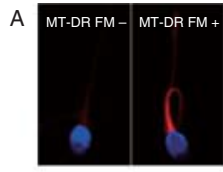

B
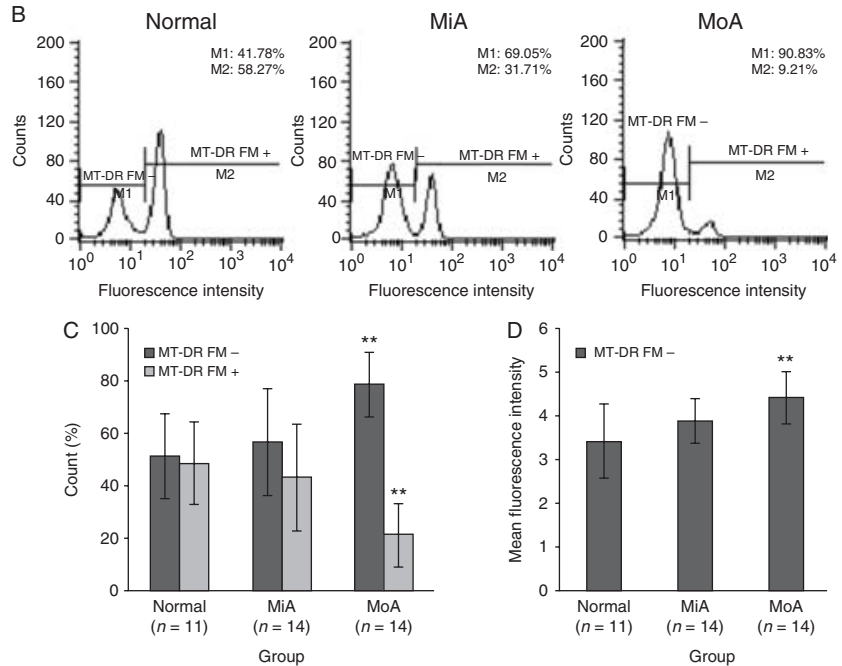

Figure 4 Mitochondrial membrane potential (MMP) analysis by flow cytometry. (A) Two subpopulations were obtained and sorted: MT-DR FM-negative spermatozoa (light unstained, left) and MT-DR FM-positive spermatozoa (deep stained, right) using a confocal laser microscope. For imaging purposes, nuclear DNA was stained with the DNA dye Hoechst 33342; (B) representative histograms obtained using flow cytometry; and (C) comparison between different group samples for the percentages of two subpopulations of spermatozoa. (D) Comparison between different group samples for the fluorescence intensity of MT-DR FM-negative spermatozoa. Data are expressed as mean \pm s.D. $n$, number of samples used. ${ }^{* *} P<0.01$ vs normal group. MiA, mild asthenozoospermia; MoA, moderate asthenozoospermia; MT-DR FM, MitoTracker Deep Red FM.

conditions, spermatozoa possess various antioxidant strategies for protecting themselves against ROS damage.

PARK7 has already been identified as a key redoxreactive protective protein implicated in the regulation of oxidative stress. According to the distribution in ejaculated spermatozoa, the surface of the posterior part of the head, the anterior part of the midpiece, and spermatozoa flagella, PARK7 was considered to play two putative roles in fertilization: one is binding to the egg, and the other is flagella movement (Yoshida et al. 2003). It has been reported that the levels of PARK7 in AS seminal plasma are significantly lower than that from healthy donors (Wang et al. 2009). In this study, we analyzed the content of PARK7 in ejaculated spermatozoa and obtained similar results showing that the levels of PARK7 in moderate AS spermatozoa were about half of those in the control. These data, together with the observation that the levels of LPO and ROS were obviously higher in the AS patients, strongly suggest that downregulation of the PARK7 protein could result in impaired antioxidant capacity of spermatozoa, which would then affect their viability and/or motility, as is observed in AS.

\section{PARK7 translocates into the damaged mitochondria to rescue the spermatozoa in $A S$}

The major finding in this study is the demonstration that PARK7 is translocated into the mitochondria of spermatozoa, where it participates in an efficient endogenous protection mechanism against mitochondrial defects.

Spermatozoa consume ATP provided by the mitochondria mainly to sustain motility, and also for various cellular events including hyperactivation, capacitation, and acrosome reaction (Ramalho-Santos et al. 2009, Pelliccione et al. 2011). In human spermatozoa, the mitochondria differ from the corresponding organelles of somatic cells in morphology. The mitochondrial sheath is organized in a helix of about 13 gyres, with two mitochondria per gyre, surrounding the axoneme at the midpiece level. Mitochondrial dysfunction is an important contributor to male infertility, including AS (Ferramosca et al. 2008). Recently, it has been demonstrated that a subpopulation of human spermatozoa showing better fertilization potential is characterized by high mitochondrial functionality (Sousa et al. 2011). In

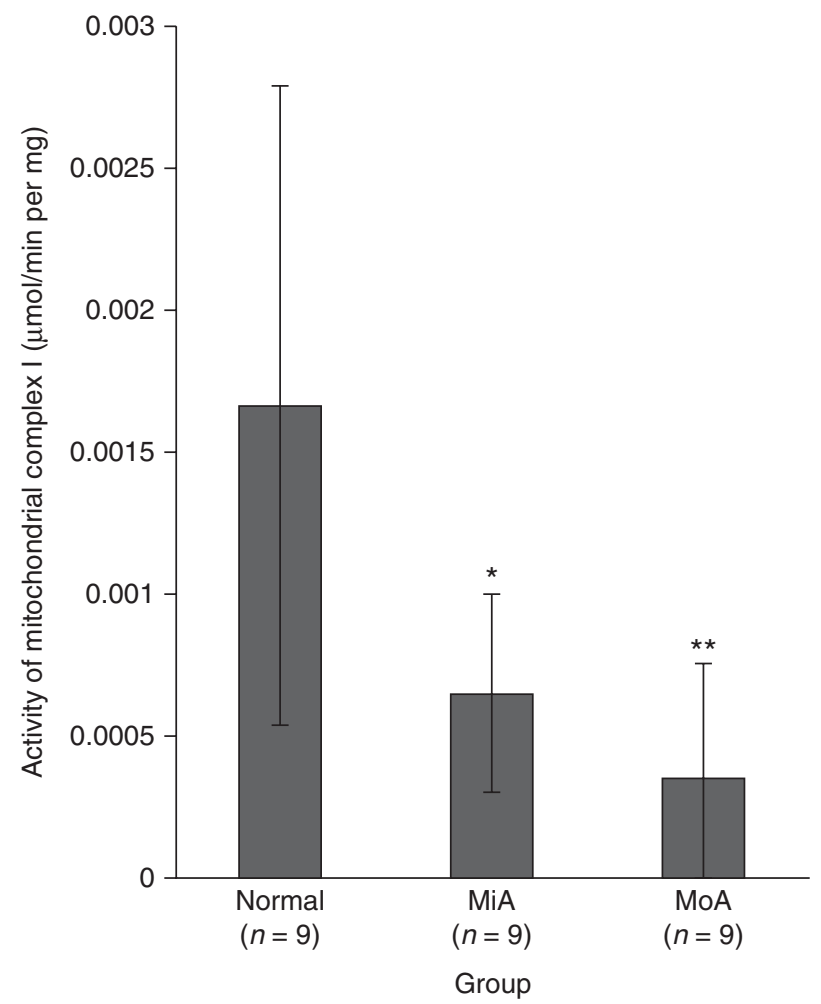

Figure 5 Impairment of mitochondrial $\mathrm{Cl}$ activity. The enzyme activity of $\mathrm{Cl}$ in sperm was measured by spectrophotometric assay. The activity of $\mathrm{Cl}$ was determined by the $\mathrm{NADH}$ content catalyzed by the enzyme in $1 \mathrm{mg}$ total protein/min at $30^{\circ} \mathrm{C}$ and $\mathrm{pH} 7.5$. Compared with the normal samples, the enzyme activity of $\mathrm{Cl}$ was significantly reduced in $\mathrm{MiA}$ and MoA samples, while a positive relationship was observed in the severity of AS. Data are expressed as mean \pm s.D. $n=9$ in each group. ${ }^{*} P<0.05$ and ${ }^{* *} P<0.01$ vs normal group. AS, asthenozoospermia; $\mathrm{MiA}$, mild asthenozoospermia; MoA, moderate asthenozoospermia. 


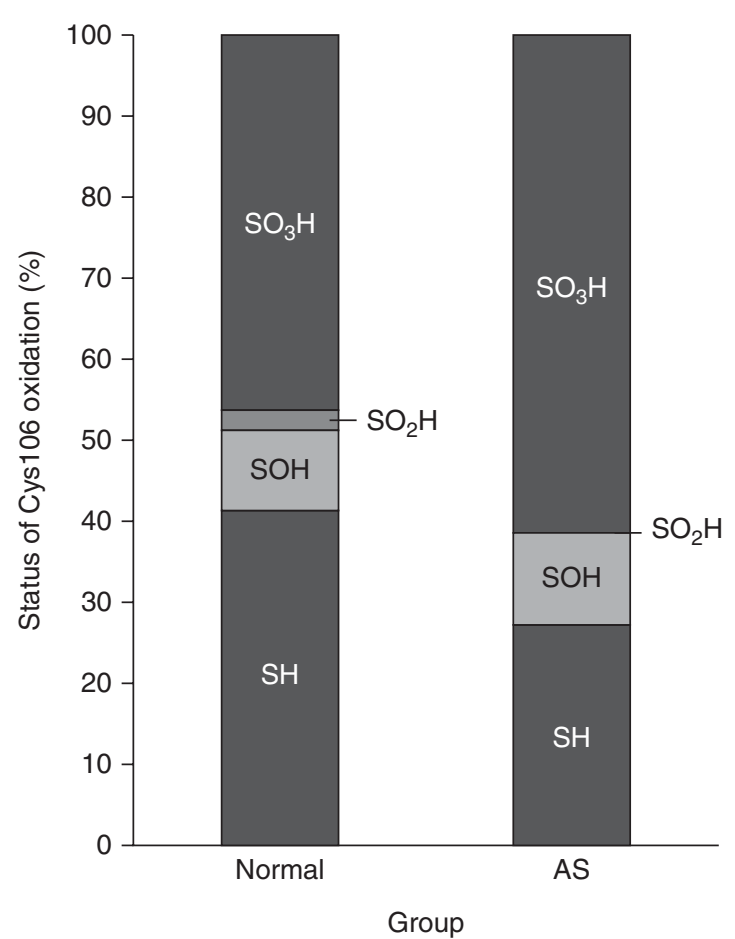

Figure 6 Cys106 oxidation modification of PARK7 by mass spectrometry assay. The $\mathrm{SO}_{3} \mathrm{H}$-modified Cys 106 form of PARK7 in AS accounted for a proportion of $61.2 \%$, compared with $46.6 \%$ for the normal form, while the sulfhydryl form decreased from 41.3 to $21.3 \%$. No significant difference in the $\mathrm{SOH}$ and $\mathrm{SO}_{2} \mathrm{H}$ forms was found between the two groups. AS, asthenozoospermia.

addition, several observations have confirmed that PARK7 is translocated from the cytoplasm into the mitochondria when MMP is decreased (Ooe et al. 2005, Junn et al. 2009, Hao et al. 2010, Krebiehl et al. 2010, Heo et al. 2012, Kaneko et al. 2013), and it maintained the $\mathrm{Cl}$ activity by directly binding to NDUFA4 and ND1, nuclear and mitochondrial DNAencoding subunits of the mitochondria respectively (Hayashi et al. 2009). Along this line, we detected the location of PARK7 by staining the spermatozoa with an anti-DJ1 antibody. The immunocytochemical results demonstrated that PARK7 appeared in the midpiece of seemingly abnormal spermatozoa, in which the mitochondria exist, and the mitochondria enriched with PARK7 showed a reduced membrane potential. However, all of these spermatozoa had high motility, because the sample was treated by density gradient centrifugation, a standard preparation technique to obtain high-motility spermatozoa samples.

To test the MMP, the mitochondria were labeled with the specific fluorescent marker, MitoTracker probe. The cell-permeant MitoTracker probes contain a mildly thiolreactive chloromethyl moiety for labeling the mitochondria. Most MitoTracker probes such as MT-DR FM, used in this study, are well retained in the mitochondria following fixation and permeabilization. It is useful to subsequently probe for other intracellular structures by immunocytochemistry. In this experiment, we found that the moderate group of AS samples possessed the least number of MT-DR FM-positive spermatozoa and the most MT-DR FM-negative spermatozoa. We also accidentally discovered that the fluorescence intensity of the MT-DR FM-negative spermatozoa in moderate AS samples was clearly higher than its counterpart in the normal samples.

The phenomenon described above could be explained as follows: without any therapeutic intervention, the translocation of PARK7 into the injured mitochondria initiated the endogenous repair mechanisms under which PARK7 improved the $\mathrm{Cl}$ activity and thus enhanced the MMP levels. Although the repair capacity of PARK7 was not sufficient to completely compensate for the decreased MMP because of the decreased amount of PARK7, it probably improved the gently injured spermatozoa motility to a certain extent. Further experiments are required to investigate these possibilities.

In addition, we examined the $\mathrm{Cl}$ activities in order to reveal the difference among AS sample groups. $\mathrm{Cl}$ is essential for oxidative phosphorylation, ROS, and electron transport system of the mitochondria for ATP production. It is highly sensitive to oxidative stress, which contributes to a range of pathologies (Wu et al. 2010). Of particular note is that we did not respectively analyze the $\mathrm{Cl}$ activity of MT-DR FM-negative and -positive spermatozoa, given that the result reflected both cell types. Reduced $\mathrm{Cl}$ activity was highly correlated with decreased expression of PARK7, further supporting the view that mitochondrial targeting of PARK7 provided significantly stronger cytoprotection.

\section{Superfluous oxidation of cysteine residues inhibits the function of PARK7}

The cysteine residues in PARK7, including Cys46, Cys53, and Cys106, appear to be very important for PARK7mediated cytoprotection. Of these, Cys106 is the major oxidation site (Canet-Aviles et al. 2004). The oxidation of Cys106 necessarily consumes ROS and thus it has been proposed that PARK7 acts as a direct scavenger of ROS (Andres-Mateos et al. 2007). Furthermore, oxidation of Cys 106 with $\mathrm{SO}_{2} \mathrm{H}$ is necessary for the mitochondrial

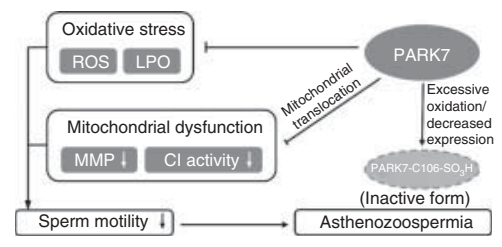

Figure 7 Proposed mechanism of PARK7 in maintaining the sperm motility. PARK7 maintains the sperm motility by protecting against oxidative stress damage and mitochondrial translocation to protect the mitochondrial function. However, PARK7 may lose these functions when oxidized excessively or when its expression is decreased. 
translocation of PARK7, but this effect is greatly diminished upon further oxidation (Canet-Aviles et al. 2004). In this study, we examined the oxidative levels of PARK7 and the results showed the enhancement of oxidation, expressed by increased $\mathrm{SO}_{3} \mathrm{H}$ residues, the highest form of oxidation, as the sperm motility decreased.

In this study, we confirmed that PARK7 is deficient in AS spermatozoa, resulting in increased LPO levels and reduced antioxidation ability of spermatozoa. We further demonstrated that under oxidative stress conditions, PARK7 was translocated into the mitochondria. In addition, we examined the oxidation levels of PARK7 protein by combined application of immunoprecipitation and LTQ Orbitrap mass spectrometry, which established a direct relationship between oxidation of PARK7 and sperm motility.

The present results provide evidence that PARK7 is closely involved in cytoprotection against ROS damage and protection of the mitochondrial function, as shown in Fig. 7, suggesting new avenues of therapeutic intervention targeting PARK7 for the treatment of AS.

\section{Supplementary data}

This is linked to the online version of the paper at http://dx.doi. org/10.1530/REP-14-0222.

\section{Declaration of interest}

The authors declare that there is no conflict of interest that could be perceived as prejudicing the impartiality of the research reported.

\section{Funding}

This work was supported by the Beijing Natural Science Foundation (no. 7122102).

\section{Acknowledgements}

The authors thank Prof. Hiroyoshi Ariga (Graduate School of Pharmaceutical Sciences, Hokkaido University, Japan) for his generous donation of anti-PARK7 polyclonal antibody and for his advice about immunoprecipitation assay technique of spermatozoa PARK7. They also thank Zhi Qu, Hanjie Jiang, Wan Li, Rutong Ren, Yingfan Yang, and Jiamin Xu for collecting semen samples.

\section{Reference}

Agarwal A, Makker K \& Sharma R 2008 Clinical relevance of oxidative stress in male factor infertility: an update. American Journal of Reproductive Immunology 59 2-11. (doi:10.1111/j.1600-0897.2007. 00559.x)

Amaral A, Ramalho-Santos J \& St John JC 2007 The expression of polymerase gamma and mitochondrial transcription factor $\mathrm{A}$ and the regulation of mitochondrial DNA content in mature human sperm. Human Reproduction 22 1585-1596. (doi:10.1093/humrep/dem030)
An CN, Jiang H, Wang Q, Yuan RP, Liu JM, Shi WL, Zhang ZY \& Pu XP 2011 Down-regulation of DJ-1 protein in the ejaculated spermatozoa from Chinese asthenozoospermia patients. Fertility and Sterility 96 19-23 (e12). (doi:10.1016/j.fertnstert.2011.04.048)

Andres-Mateos E, Perier C, Zhang L, Blanchard-Fillion B, Greco TM, Thomas B, Ko HS, Sasaki M, Ischiropoulos H, Przedborski S et al. 2007 DJ-1 gene deletion reveals that DJ-1 is an atypical peroxiredoxin-like peroxidase. PNAS 104 14807-14812. (doi:10.1073/pnas.0703219104)

Bonifati V, Rizzu P, van Baren MJ, Schaap O, Breedveld GJ, Krieger E, Dekker MC, Squitieri F, Ibanez P, Joosse M et al. 2003 Mutations in the DJ-1 gene associated with autosomal recessive early-onset parkinsonism. Science 299 256-259. (doi:10.1126/science.1077209)

Canet-Aviles RM, Wilson MA, Miller DW, Ahmad R, McLendon C, Bandyopadhyay S, Baptista MJ, Ringe D, Petsko GA \& Cookson MR 2004 The Parkinson's disease protein DJ-1 is neuroprotective due to cysteine-sulfinic acid-driven mitochondrial localization. PNAS 101 9103-9108. (doi:10.1073/pnas.0402959101)

Chemes HE, Olmedo SB, Carrere C, Oses R, Carizza C, Leisner M \& Blaquier J 1998 Ultrastructural pathology of the sperm flagellum: association between flagellar pathology and fertility prognosis in severely asthenozoospermic men. Human Reproduction 13 2521-2526. (doi:10.1093/humrep/13.9.2521)

Curi SM, Ariagno JI, Chenlo PH, Mendeluk GR, Pugliese MN, Sardi Segovia LM, Repetto HE \& Blanco AM 2003 Asthenozoospermia: analysis of a large population. Archives of Andrology 49 343-349. (doi:10.1080/713828220)

Ferramosca A, Focarelli R, Piomboni P, Coppola L \& Zara V 2008 Oxygen uptake by mitochondria in demembranated human spermatozoa: a reliable tool for the evaluation of sperm respiratory efficiency. International Journal of Andrology 31 337-345. (doi:10.1111/j.13652605.2007.00775.x)

Gao JW, Yamane T, Maita H, Ishikawa S, Iguchi-Ariga SM, Pu XP \& Ariga H 2011 DJ-1-mediated protective effect of protocatechuic aldehyde against oxidative stress in SH-SY5Y cells. Journal of Pharmacological Sciences 115 36-44. (doi:10.1254/jphs.10271FP)

Hao LY, Giasson BI \& Bonini NM 2010 DJ-1 is critical for mitochondrial function and rescues PINK1 loss of function. PNAS 107 9747-9752. (doi:10.1073/pnas.0911175107)

Hayashi T, Ishimori C, Takahashi-Niki K, Taira T, Kim YC, Maita H, Maita C, Ariga H \& Iguchi-Ariga SM 2009 DJ-1 binds to mitochondrial complex I and maintains its activity. Biochemical and Biophysical Research Communications 390 667-672. (doi:10.1016/j.bbrc. 2009.10.025)

Heo JY, Park JH, Kim SJ, Seo KS, Han JS, Lee SH, Kim JM, Park JI, Park SK, Lim K et al. 2012 DJ-1 null dopaminergic neuronal cells exhibit defects in mitochondrial function and structure: involvement of mitochondrial complex I assembly. PLOS ONE 7 e32629. (doi:10.1371/journal.pone. 0032629)

Junn E, Jang WH, Zhao X, Jeong BS \& Mouradian MM 2009 Mitochondrial localization of DJ-1 leads to enhanced neuroprotection. Journal of Neuroscience Research 87 123-129. (doi:10.1002/jnr.21831)

Kaneko Y, Tajiri N, Shojo H \& Borlongan CV 2013 Oxygen-glucosedeprived rat primary neural cells exhibit DJ-1 translocation into healthy mitochondria: a potent stroke therapeutic target. CNS Neuroscience \& Therapeutics 20 275-281. (doi:10.1111/cns.12208)

Kinumi T, Kimata J, Taira T, Ariga H \& Niki E 2004 Cysteine-106 of DJ-1 is the most sensitive cysteine residue to hydrogen peroxide-mediated oxidation in vivo in human umbilical vein endothelial cells. Biochemical and Biophysical Research Communications 317 722-728. (doi:10.1016/ j.bbrc.2004.03.110)

Klinefelter GR, Laskey JW, Ferrell J, Suarez JD \& Roberts NL 1997 Discriminant analysis indicates a single sperm protein (SP22) is predictive of fertility following exposure to epididymal toxicants. Journal of Andrology 18 139-150. (doi:10.1002/j.1939-4640.1997.tb01894.x)

Klinefelter GR, Welch JE, Perreault SD, Moore HD, Zucker RM, Suarez JD, Roberts NL, Bobseine K \& Jeffay S 2002 Localization of the sperm protein SP22 and inhibition of fertility in vivo and in vitro. Journal of Andrology 23 48-63.

Koppers AJ, De Iuliis GN, Finnie JM, McLaughlin EA \& Aitken RJ 2008 Significance of mitochondrial reactive oxygen species in the generation of oxidative stress in spermatozoa. Journal of Clinical Endocrinology and Metabolism 93 3199-3207. (doi:10.1210/jc.2007-2616) 
Krebiehl G, Ruckerbauer S, Burbulla LF, Kieper N, Maurer B, Waak J, Wolburg H, Gizatullina Z, Gellerich FN, Woitalla D et al. 2010 Reduced basal autophagy and impaired mitochondrial dynamics due to loss of Parkinson's disease-associated protein DJ-1. PLOS ONE 5 e9367. (doi:10.1371/journal.pone.0009367)

Mannella CA 2008 Structural diversity of mitochondria: functional implications. Annals of the New York Academy of Sciences 1147 171-179. (doi:10.1196/annals.1427.020)

Mitsumoto A \& Nakagawa Y 2001 DJ-1 is an indicator for endogenous reactive oxygen species elicited by endotoxin. Free Radical Research 35 885-893. (doi:10.1080/10715760100301381)

Mitsumoto A, Nakagawa Y, Takeuchi A, Okawa K, Iwamatsu A \& Takanezawa Y 2001 Oxidized forms of peroxiredoxins and DJ-1 on two-dimensional gels increased in response to sublethal levels of paraquat. Free Radical Research 35 301-310. (doi:10.1080/ 10715760100300831)

Mundy AJ, Ryder TA \& Edmonds DK 1995 Asthenozoospermia and the human sperm mid-piece. Human Reproduction 10 116-119. (doi:10. 1093/humrep/10.1.116)

Nagakubo D, Taira T, Kitaura H, Ikeda M, Tamai K, Iguchi-Ariga SM \& Ariga H 1997 DJ-1, a novel oncogene which transforms mouse NIH3T3 cells in cooperation with ras. Biochemical and Biophysical Research Communications 231 509-513. (doi:10.1006/ bbrc.1997.6132)

Okada M, Matsumoto K, Niki T, Taira T, Iguchi-Ariga SM \& Ariga H 2002 DJ-1, a target protein for an endocrine disrupter, participates in the fertilization in mice. Biological \& Pharmaceutical Bulletin 25 853-856. (doi:10.1248/bpb.25.853)

Ooe H, Taira T, Iguchi-Ariga SM \& Ariga H 2005 Induction of reactive oxygen species by bisphenol $\mathrm{A}$ and abrogation of bisphenol A-induced cell injury by DJ-1. Toxicological Sciences 88 114-126. (doi:10.1093/ toxsci/kfi278)

WHO WHO Laboratory Manual for the Examination and Processing of Human Semen 5th ed. WHO library cataloguing - World Health Organization 2010, Geneva, Switzerland.

Pelliccione F, Micillo A, Cordeschi G, D'Angeli A, Necozione S, Gandini L, Lenzi A, Francavilla F \& Francavilla S 2011 Altered ultrastructure of mitochondrial membranes is strongly associated with unexplained asthenozoospermia. Fertility and Sterility 95 641-646. (doi:10.1016/j. fertnstert.2010.07.1086)

Ramalho-Santos J, Varum S, Amaral S, Mota PC, Sousa AP \& Amaral A 2009 Mitochondrial functionality in reproduction: from gonads and gametes to embryos and embryonic stem cells. Human Reproduction Update 15 553-572. (doi:10.1093/humupd/dmp016)

Ruiz-Pesini E, Diez C, Lapena AC, Perez-Martos A, Montoya J, Alvarez E, Arenas J \& Lopez-Perez MJ 1998 Correlation of sperm motility with mitochondrial enzymatic activities. Clinical Chemistry $\mathbf{4 4}$ 1616-1620.

Ruiz-Pesini E, Lapena AC, Diez C, Alvarez E, Enriquez JA \& Lopez-Perez MJ 2000 Seminal quality correlates with mitochondrial functionality. Clinica Chimica Acta 300 97-105. (doi:10.1016/S0009-8981(00)00305-3)

Searle AJ \& Willson RL 1983 Stimulation of microsomal lipid peroxidation by iron and cysteine. Characterization and the role of free radicals. Biochemical Journal 212 549-554.
Shinbo Y, Niki T, Taira T, Ooe H, Takahashi-Niki K, Maita C, Seino C, Iguchi-Ariga SM \& Ariga H 2006 Proper SUMO-1 conjugation is essential to DJ-1 to exert its full activities. Cell Death and Differentiation 13 96-108. (doi:10.1038/sj.cdd.4401704)

Sikka SC, Rajasekaran M \& Hellstrom WJ 1995 Role of oxidative stress and antioxidants in male infertility. Journal of Andrology 16 464-468. (doi:10.1002/j.1939-4640.1995.tb00566.x)

Sousa AP, Amaral A, Baptista M, Tavares R, Caballero Campo P, Caballero Peregrin P, Freitas A, Paiva A, Almeida-Santos T \& Ramalho-Santos J 2011 Not all sperm are equal: functional mitochondria characterize a subpopulation of human sperm with better fertilization potential. PLoS ONE 6 e18112. (doi:10.1371/journal.pone.0018112)

Taira T, Saito Y, Niki T, Iguchi-Ariga SM, Takahashi K \& Ariga H 2004 DJ-1 has a role in antioxidative stress to prevent cell death. EMBO Reports $\mathbf{5}$ 213-218. (doi:10.1038/sj.embor.7400074)

Takahashi-Niki K, Niki T, Taira T, Iguchi-Ariga SM \& Ariga H 2004 Reduced anti-oxidative stress activities of DJ-1 mutants found in Parkinson's disease patients. Biochemical and Biophysical Research Communications 320 389-397. (doi:10.1016/j.bbrc.2004.05.187)

Talevi R, Barbato V, Fiorentino I, Braun S, Longobardi S \& Gualtieri R 2013 Protective effects of in vitro treatment with zinc, D-aspartate and coenzyme q10 on human sperm motility, lipid peroxidation and DNA fragmentation. Reproductive Biology and Endocrinology 11 81. (doi:10. 1186/1477-7827-11-81)

Wagenfeld A, Yeung CH, Strupat K \& Cooper TG 1998 Shedding of a rat epididymal sperm protein associated with infertility induced by ornidazole and $\boldsymbol{\alpha}$-chlorohydrin. Biology of Reproduction $\mathbf{5 8}$ 1257-1265. (doi:10.1095/biolreprod58.5.1257)

Wang J, Zhang HR, Shi HJ, Ma D, Zhao HX, Lin B \& Li RS 2009 Proteomic analysis of seminal plasma from asthenozoospermia patients reveals proteins that affect oxidative stress responses and semen quality. Asian Journal of Andrology 11 484-491. (doi:10.1038/ aja.2009.26)

Welch JE, Barbee RR, Roberts NL, Suarez JD \& Klinefelter GR 1998 SP22: a novel fertility protein from a highly conserved gene family. Journal of Andrology 19 385-393. (doi:10.1002/j.1939-4640.1998.tb02029.x)

Wu H, Xing K \& Lou MF 2010 Glutaredoxin 2 prevents $\mathrm{H}(2) \mathrm{O}(2)$-induced cell apoptosis by protecting complex I activity in the mitochondria. Biochimica et Biophysica Acta 1797 1705-1715. (doi:10.1016/j.bbabio. 2010.06.003)

Yokota T, Sugawara K, Ito K, Takahashi R, Ariga H \& Mizusawa H 2003 Down regulation of $D$ J-1 enhances cell death by oxidative stress, ER stress, and proteasome inhibition. Biochemical and Biophysical Research Communications 312 1342-1348. (doi:10.1016/j.bbrc.2003.11.056)

Yoshida K, Sato Y, Yoshiike M, Nozawa S, Ariga H \& Iwamoto T 2003 Immunocytochemical localization of DJ-1 in human male reproductive tissue. Molecular Reproduction and Development 66 391-397. (doi:10.1002/mrd.10360)

Received 23 April 2014

First decision 2 June 2014

Revised manuscript received 4 June 2014

Accepted 11 June 2014 